\title{
Algunas relaciones entre investigación, práctica pedagógica y formación inicial: relatos de una experiencia.
}

Por: Amórtegui Elías ${ }^{1} \&$ Valbuena Ussa Edgar ${ }^{2}$

\section{INTRODUCCIÓN}

Desde la perspectiva del Proyecto Curricular de Licenciatura en Biología (PCLB), la Investigación se convierte en un elemento de vital importancia en la formación inicial de profesores de Biología, desde una mirada crítica y transformadora que repercute en el diseño y ejecución de los "Proyectos de práctica pedagógica" y posteriormente en los Trabajos de Grado.

Es importante cuestionarnos entonces ¿Qué favorece dicha relación?, ¿Qué factores lo obstaculizan?, ¿Qué aporta a nuestra formación como futuros docentes? Lo anterior a partir de la experiencia en la participación de las VIII Jornadas Nacionales y III Congreso Internacional de Enseñanza de la Biología llevado a cabo en Mar del Plata, con la ponencia titulada "Las prácticas de campo y el Conocimiento Profesional del Profesor de Biología en su formación inicial".

\section{SOBRE LA TEORÍA}

A través del tiempo la profesión docente ha perdido validez y se ha subestimado (social y epistemológicamente) la labor de los maestros al considerar que para enseñar, sólo es necesario manejar el conocimiento disciplinar a profundidad, dejando de lado el hecho de que como profesionales de la educación, se posee un conocimiento particular que aparte de incluir lo disciplinar, posibilita procesos de enseñanza diferentes a como lo pueden realizar los profesionales de otras áreas (Valbuena, 2007a).

Actualmente se están realizando investigaciones que contribuyen a ampliar la producción acerca del Conocimiento Profesional del Profesor, como resultado de las mismas se plantea la importancia de investigar en el ámbito de la formación docente, los procesos que promueven la construcción de dicho conocimiento y su vinculación en la formación inicial de maestros (Tardif, 2004).

${ }^{1}$ Estudiante de Décimo semestre de Licenciatura en Biología de la Universidad Pedagógica Nacional. Tesista y Monitor de Investigación del Grupo Conocimiento Profesional del Profesor de Ciencias. folkerpan@hotmail.com

2 Profesor Departamento de Biología, Universidad Pedagógica Nacional y Coordinador de la Línea de Investigación Conocimiento Profesional del Profesor de Ciencias.

edgarorlay@hotmail.com 
La investigación en la Universidad Pedagógica Nacional (UPN) ha sido reconocida como eje transversal en la formación de maestros, siendo un pilar que orienta la construcción de sus planes curriculares, teniendo en cuenta que al revisar los objetivos planteados en el Proyecto Político y Pedagógico (PPP) de la UPN, se encuentra que “...pretende formar y cualificar docentes, producir conocimientos y fortalecer la identidad profesional del educador, su valoración y proyección en el contexto social como un trabajador de la cultura..." (Proyecto Político Pedagógico De La Universidad Pedagógica Nacional, 2004).

En concordancia con lo anterior, el Proyecto Curricular de Licenciatura en Biología (PCLB), ha planteado la investigación como un reto que debe ser pensado y asumido por los futuros maestros, pues se confía que desde la formación inicial se contribuya a la construcción del Conocimiento Profesional, lo cual representa una ventaja ya que le permite a los futuros licenciados, adquirir habilidades no solo conceptuales y actitudinales, sino también de investigación.

El PCLB, tiene como ejes transversales “... la formación en el desarrollo humano integral, la investigación y la formación pedagógica y didáctica...".

Dentro de la misión del Departamento de Biología se plantea como principio, “...la pertinencia y rigurosidad de la investigación del más alto nivel acerca de los problemas y necesidades nacionales en el ámbito de su competencia..." (PCLB, p. 17).

Además dentro de los objetivos del Departamento, se plantea “...diseñar y ejecutar proyectos de investigación en educación y en pedagogía de la Biología y saberes afines..." (PCLB, p. 19).

El PCLB, es considerado un proyecto de investigación educativa, de tal manera que se asume como un proceso formativo sistemático, en el cual se propende por el desarrollo de habilidades y competencias que permiten al Licenciado en Biología, el logro de los objetivos propuestos. Con estos objetivos, el futuro maestro debe desarrollar habilidades de tipo investigativas, que implican el dominio de conocimientos de un área del saber, el dominio de los procedimientos científicos y el razonamiento complejo.

Bajo toda esta perspectiva e ideas sobre la investigación, y su importancia en el ámbito educativo y en la formación inicial de maestros, es importante cuestionarnos acerca de ¿Qué aspectos favorecen la investigación?, ¿Qué factores lo obstaculizan?, ¿Con qué se relaciona dicha investigación?, ¿Qué aporta a nuestra formación como futuros docentes?

Desde mi experiencia, podría afirmar que uno de los primeros elementos importantes en el desarrollo investigativo en la formación inicial, es el proyecto que se desarrolla en sexto semestre. El eje curricular de sexto semestre es bastante particular en la dinámica de la formación académica, pues al ser el cierre del ciclo de fundamentación, el futuro docente debe haber alcanzado los objetivos planteados para este ciclo y así poder ingresar al ciclo de profundización. 
Dicho proyecto de semestre, al término del ciclo de fundamentación, apunta a indagar si se han alcanzado los logros formativos presupuestados. Por esta razón, principalmente, se debe orientar su realización para que los estudiantes puedan plasmar en una propuesta sus desarrollos conceptuales, procedimentales y actitudinales con relación a la investigación (pedagógica, didáctica y disciplinar), y sus expectativas profesionales que se empezarán a esbozar en exteriorización de sus intencionalidades en su Práctica Docente, su Proyecto de grado y su futuro profesional (Sierra, 2007).

\section{Proyecto Curricular de Licenciatura en Biología}

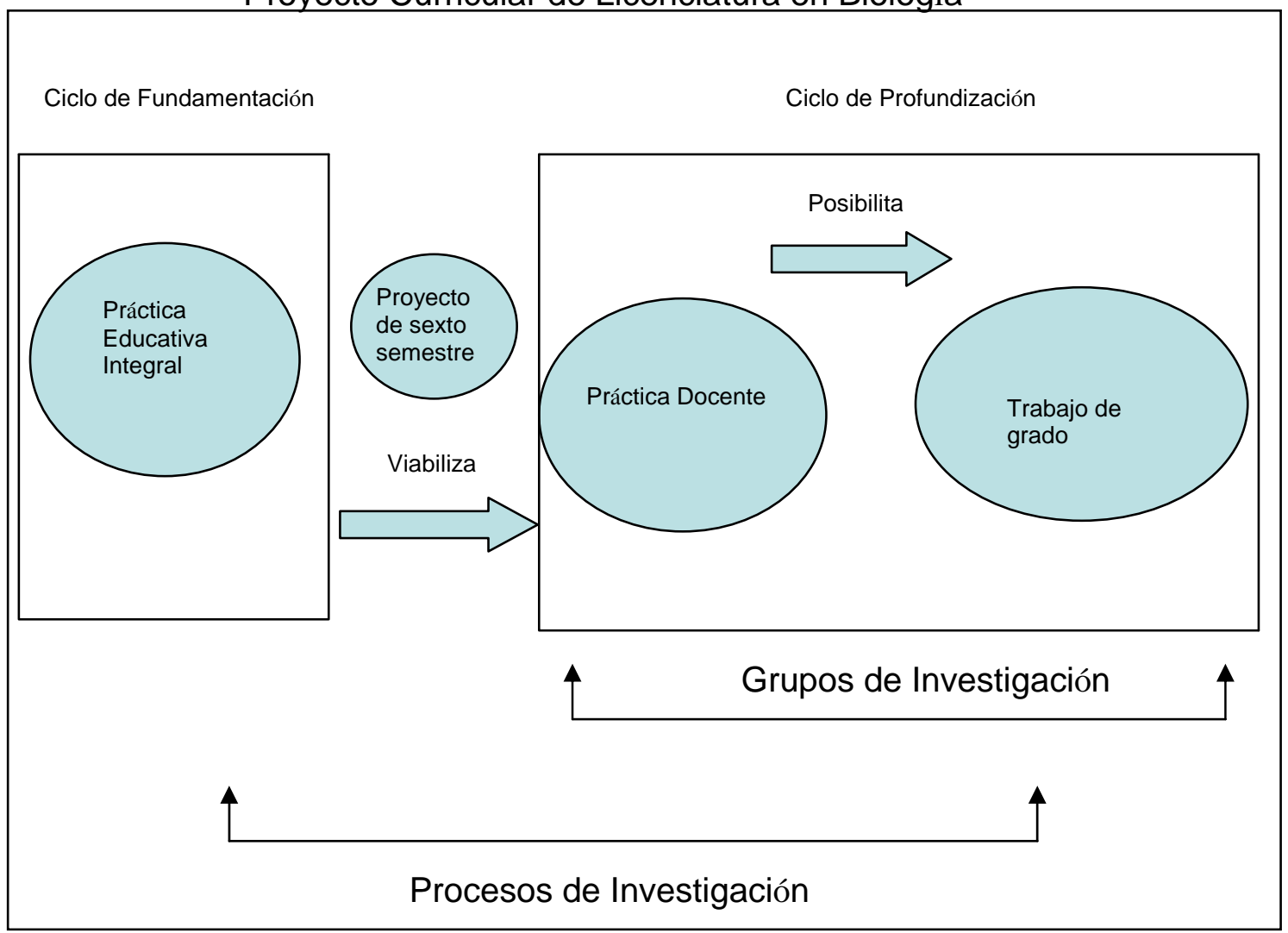

Gráfica 1: Dinámica investigativa en el Proyecto Curricular de Licenciatura en Biología

Al comenzar su proceso de formación, el futuro licenciado se desenvuelve en el Ciclo de Fundamentación (Ver gráfica 1) durante los seis primeros semestres. Durante estos desarrolla una actividad de integración de los espacios académicos denominado "Proyecto de Semestre"; además realiza su trabajo de Práctica Educativa Integral, en donde tiene la posibilidad desde el primer semestre, de reflexionar y estar en contacto con la realidad educativa a partir de experiencias particulares. Esta Práctica, constituye la base de formación para la consolidación y desarrollo de la Práctica Docente y el Trabajo de grado.

Luego de finalizar el Ciclo de Fundamentación, el futuro maestro ingresa al Ciclo de Profundización a desarrollar su Práctica Docente dentro de uno de los Grupos de Investigación que trabajan en el Departamento de Biología, y con base en el proyecto elaborado en sexto semestre, desarrollar su práctica (Ver gráfica 1). 
Dicha práctica docente se realiza a través de proyectos pedagógicos que identifiquen problemáticas educativas y en donde los futuros maestros propongan alternativas para abordar dichas problemáticas (PCLB, p 71).

Actualmente existen en el Departamento de Biología, la posibilidad de ingresar a nueve Grupos de Investigación y allí realizar tanto prácticas docentes como trabajos de grado (Valbuena, 2007b).

A diferencia del Ciclo de Fundamentación, el Ciclo de Profundización hace explicito en sus objetivos, que el estudiante, desarrolle proyectos investigativos, además debe planear, diseñar, aplicar, administrar, evaluar y divulgar dichos proyectos (PCLB, p. 95).

En este sentido, es en el Ciclo de Profundización, en donde el futuro maestro pone en juego en mayor medida sus habilidades investigativas y toma un papel activo en el planteamiento de investigaciones, desde una perspectiva mucho más rigurosa, teniendo en cuenta objetivos, metodologías, antecedentes, entre otros, que hacen parte de su formación como futuro docente.

\section{SOBRE LA PRÁCTICA}

Teniendo en cuenta todos los aspectos teóricos sobre la investigación en el PCLB, Los Ciclos de Fundamentación y Profundización, los Grupos de Investigación, las Prácticas docentes y el Trabajo de grado, mencionados anteriormente quisiera en este apartado, mostrar cómo ha sido mi formación investigativa durante el cierre del ciclo de fundamentación y durante mi finalización del ciclo de profundización y las implicaciones que ello ha tenido sobre mi formación profesional.

Durante mi paso por sexto semestre, propuse como proyecto de semestre indagar sobre las Habilidades y Competencias Propiciadas por las Prácticas de Campo en los estudiantes del Ciclo de Fundamentación de La Universidad Pedagógica Nacional. Un factor clave que dinamiza el planteamiento de este proyecto es la denominada "matriz" que se elabora en la primera entrega del proyecto de sexto semestre, en donde el estudiante debe con base en aspectos como los ejes curriculares, organizar e identificar sus tendencias formativas; esto se lleva a cabo haciendo la revisión de documentos (ensayos, proyectos, salidas de campo, entre otros) elaborados durante el desarrollo del ciclo de fundamentación explicitando los aportes positivos y negativos de estos (Sierra, 2007). Esto permite esbozar el proyecto de semestre a partir de los intereses y necesidades del futuro profesor.

Ya en el ciclo de profundización ingresé al Grupo de Investigación Conocimiento Profesional del Profesor de Ciencias, coordinado por el profesor Edgar Valbuena. En este colectivo, presenté mi propuesta de sexto semestre y a partir de discusiones y recomendaciones y dado el contexto de mi práctica, postulé como proyecto de práctica 1 el trabajo La Práctica de Campo como contribuyente a la construcción del Conocimiento Profesional Del Profesor De Biología en estudiantes de formación Inicial, el cual durante la práctica 2 se transformó en la propuesta Contribución a la Construcción del Conocimiento Profesional del Profesor de Biología, mediante la 
apropiación de la Práctica de Campo como estrategia en la enseñanza de la Biología en estudiantes de formación inicial.

Durante la práctica 1 y 2 es fundamental el trabajo con los asesores y tutores, pues a partir de los resultados hallados, los instrumentos aplicados, la fundamentación teórica, y primordialmente, los intereses del futuro profesor, ellos guían el óptimo desarrollo de los proyectos de práctica, en donde los intereses del estudiante en formación deben ser claros y explícitos de tal manera que el proyecto de práctica sea algo propio que parta de los deseos y gustos educativos.

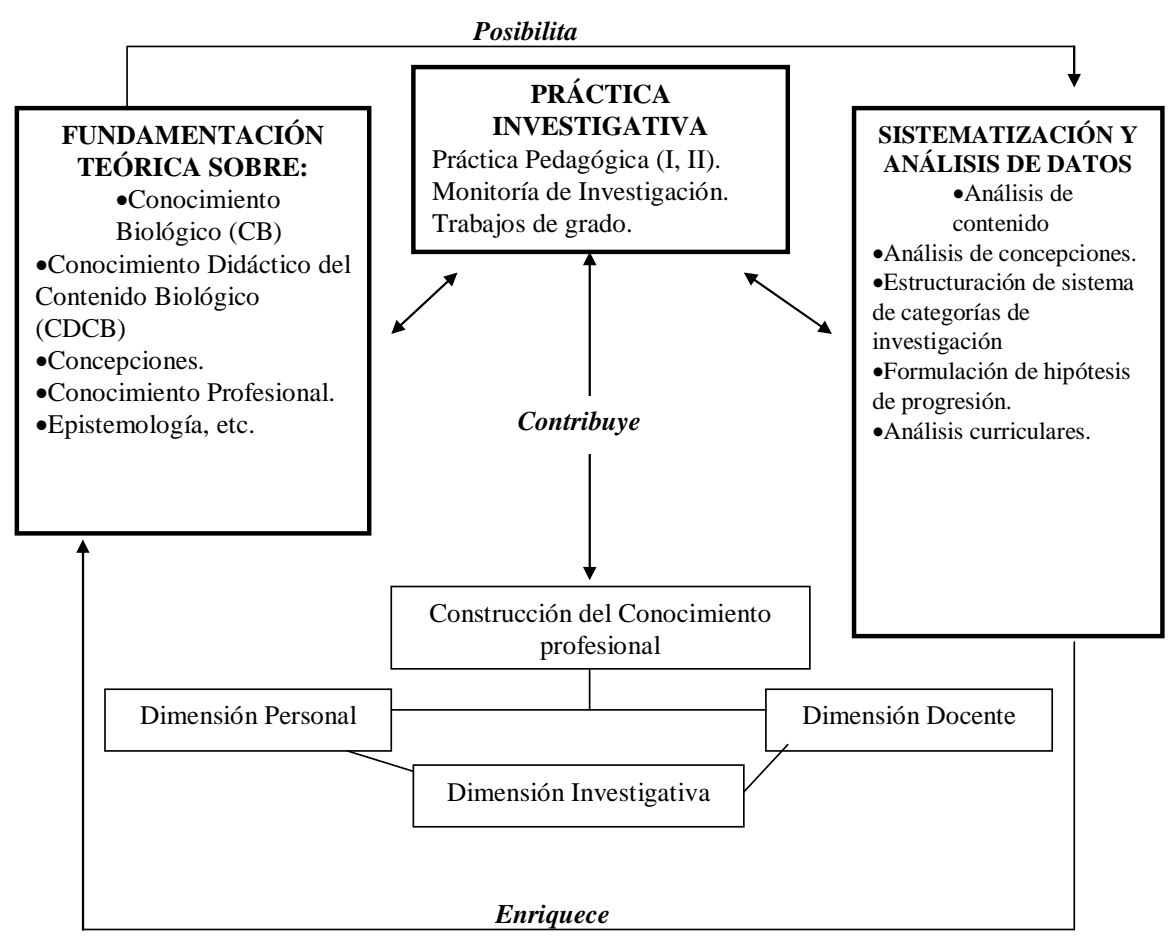

Gráfica 2: Dinámica de Investigación del Grupo Conocimiento Profesional del Profesor de Ciencias.

El aspecto que más ha favorecido mi formación investigativa, es el Grupo de Investigación en el cual me encuentro. La dinámica al interior de dicho grupo, se orienta desde tres escenarios: la práctica investigativa, los seminarios semanales de fundamentación teórica y los de sistematización (Ver gráfica 2). Algunas actividades que se realizan en el grupo son la producción de escritos, la socialización de resultados, la búsqueda de material bibliográfico, el diseño de instrumentos para obtención de datos y la participación en eventos de carácter nacional e internacional.

En el escenario de práctica investigativa, se implementa la práctica pedagógica 1 y 2 , las monitorias de investigación (opcional) y trabajos de grado; éste escenario se complementa 
a partir de los seminarios semanales en los cuales se fortalece la fundamentación teórica, generando discusiones frente al Conocimiento Biológico (CB) y el Conocimiento Didáctico del Contenido Biológico (CDCB), entre otros, lo cual posibilita el proceso de sistematización y análisis de los datos obtenidos durante los proyectos de investigación del grupo (Amórtegui, E; Bandera D; Bernal, C; Correa, M; Pachón S; Valbuena, E. 2008). Cabe resaltar que la reflexión juega un papel fundamental en la formación, pues permite ir evaluando y repensando los aspectos que constituyen dicha formación, con el fin de proyectar una mejor labor docente, ya que la formación de maestros debe abogar porque éstos se reconozcan como sujetos de conocimientos propios de su profesión. La formación del profesorado debe partir de un enfoque reflexivo, en el que se analicen las prácticas profesionales, las tareas y los conocimientos de los maestros (Tardif, 2004).

La dinámica del Grupo de Investigación tiene gran influencia en mi formación profesional, ya que actividades como la formulación de problemas de investigación, la elaboración de informes, la realización de relatorías, el diseño de instrumentos de investigación, la obtención de datos, la sistematización y análisis de los mismos, nutren las prácticas pedagógicas y los trabajos de grado, aportando elementos para su buen desarrollo (Amórtegui, E; Bernal, C; Correa, M; Valbuena, E. 2008).

En este sentido, el Grupo de Investigación reconoce que es fundamental la formación de profesores de Biología, desde el enfoque del profesor investigador, en donde es importante que el futuro maestro sea capaz de cuestionar y reestructurar sus teorías implícitas y las rutinas aprendidas en su experiencia como estudiantes y que sea además de ser un profesional autónomo, crítico e investigador y un profesional reflexivo sobre su propia práctica (García, 2006).

Por otra parte, un aspecto relevante del Grupo, es la divulgación de los trabajos que se realizan de prácticas pedagógicas y de trabajos de grado. Como ejemplo cito mi participación en las VIII Jornadas Nacionales y III Congreso Internacional de Enseñanza de la Biología, realizado en Mar del Plata en el presenta año. En dicho evento presenté el trabajo en compañía de dos maestros titulado Las prácticas de campo y el conocimiento profesional del profesor de biología en su formación inicial. Dicho trabajo presentaba diversos aspectos de los proyectos de práctica pedagógica 1 y 2 desarrollados durante el año 2007 y 2008. Con esta participación se logró socializar frente a la comunidad de especialistas a nivel latinoamericano, algunas de las experiencias y reflexiones que he realizado junto con el Grupo de Investigación.

Esta participación fue de vital importancia para mi formación, pues me permitió el intercambio y socialización de experiencias realizadas en diversos contextos, diversos países, diversas situaciones, no solo en la formación permanente sino también en la formación inicial de maestros, lo cual repercute y retroalimenta los procesos formativos que se llevan a cabo en el DBI y en la Universidad Pedagógica Nacional.

En general dicha situación corresponde más a la excepción que a la regla, pues los valiosos proyectos de práctica que realizan los estudiantes de biología muy pocas veces son 
divulgados, inclusive al interior del Departamento de Biología, con lo cual no se estaría socializando frente al colectivo de maestros y estudiantes, todas las propuestas, problemáticas, estrategias, instrumentos, y en general experiencias que se suscitan con el desarrollo de las prácticas pedagógicas.

Finalmente cabe aclarar que siguiendo la línea investigativa del PCLB, lo que antes fue mi proyecto de semestre, luego mi práctica pedagógica 1 y 2 , ahora sigue dando frutos en el trabajo de grado que actualmente desarrollo, titulado Caracterización de las prácticas de campo en el ciclo de fundamentación del Proyecto Curricular de Licenciatura en Biología de la Universidad Pedagógica Nacional, en el marco de la construcción del Conocimiento Profesional del Profesor de Biología.

\section{CONCLUSIONES}

Este escrito permite concluir algunos aspectos, no a manera de absolutismos, sino por el contrario de seguir planteando el debate en el contexto educativo.

El Proyecto Curricular de Licenciatura en Biología se caracteriza por tener una perspectiva investigativa para la formación de futuros profesores de biología, en donde se concibe la investigación como aquella que permite la integración del Ciclo de Fundamentación con el Ciclo de Profundización, en cuanto al desarrollo de prácticas pedagógicas y trabajos de grado.

La investigación en la formación inicial de maestros, en sus diversos contextos (proyectos de práctica, trabajos de grado, monitorias), es un elemento transversal en dicha formación, que permite la constitución de un sujeto maestro investigador crítico, que le permite ser consciente de las particularidades de su conocimiento profesional.

Entre los diferentes factores que inciden en la formación investigativa de los futuros docentes, el Grupo de Investigación es uno de los más fundamentales, pues en él se desarrolla todas las actividades investigativas, guiadas, socializadas y discutidas entre el colectivo de maestros y estudiantes.

Es importante resaltar la importancia de la escritura y la divulgación de los proyectos que desarrollan los futuros maestros, pues esto permite dar a conocer las experiencias que se realizan en el PCLB y además permite la constitución de un ser maestro investigativo y comprometido con la producción de conocimiento.

\section{Bibliografía}

- AMÓRTEGUI, E; BANDERA D; BERNAL, C; CORREA, M; PACHÓN S; VALBUENA, E. 2008. Construcción del conocimiento profesional del profesor de biología, en el contexto de la investigación en la formación inicial. Memorias. V Encuentro nacional de experiencias en enseñanza de la biología y la educación ambiental. 1-9 p. Bogotá D.C. 
- AMÓRTEGUI, E; BERNAL, C; CORREA, M; VALBUENA, E. 2008. Construcción del conocimiento profesional del profesor de biología, en el contexto de la investigación en formación inicial. Memorias. V Encuentro iberoamericano de colectivos y redes de maestros que hacen investigación e innovación desde su escuela y comunidad. Caracas.

- GARCÍA, E 2006. La integración de la teoría con la práctica en la formación inicial del profesorado. En Alambique: Didáctica de las Ciencias Experimentales. 47. p 65-73.

- Proyecto Político Pedagógico de la Universidad Pedagógica Nacional 2004. ARFO Editores. Bogotá D.C. $99 \mathrm{p}$

- Proyecto Curricular de Licenciatura en Biología. 1999. Universidad Pedagógica Nacional. Documento de referencia. Bogotá. D.C. 150 p.

- SIERRA, Carlos 2007. Reflexiones en torno al proyecto de semestre del eje curricular interacción. Documento inédito.

- TARDIF, Maurice. 2004. Los saberes del docente y su desarrollo profesional. Editorial Narcea: Madrid. $234 \mathrm{p}$.

- VAlBUENA, E. 2007a. El Conocimiento Didáctico del Contenido Biológico. Estudio de las concepciones disciplinares y didácticas de futuros docentes de la Universidad Pedagógica Nacional (Colombia). Tesis para optar al título de Doctor. Universidad Complutense de Madrid. Madrid. 633 p.

- VALBUENA, Edgar 2007 Compilador. Grupos, Líneas y Proyectos de Investigación. Documento Inédito. Bogotá D.C. Junio. 59 p. 